\title{
LIDERAZGO TRANSFORMACIONAL Y GÉNERO EN HOTELES DE 4 Y 5 ESTRELLAS DE TIJUANA (MÉXICO)
}

\author{
Isis Arlene Díaz Carrión \\ Ma. Cruz Lozano Ramírez. \\ Julio César Montiel Flores \\ Universidad Autónoma de Baja California. México
}

\section{RESUMEN}

Esta investigación descriptiva, que aplica la técnica de entrevista en profundidad semi estructurada, muestra las interacciones de los estilos de liderazgo y el género en hoteles de 4 y 5 Estrellas de Tijuana, México. Los resultados reflejan reconocimiento de la calidad del liderazgo de las mujeres, así como las interrelaciones entre estereotipos de género y liderazgo transformacional como consecuencia de la carga de trabajo de cuidados. Las gerentes prefieren un estilo de liderazgo participativo como estrategia para formar alianzas y evitar cuestionamientos sobre su capacidad de liderazgo, y como estrategia de conciliación del trabajo y la familia.

Palabras clave: género; liderazgo; hoteles; Tijuana.

\section{Transformational leadership and gender in 4 and 5 star hotels in Tijuana (Mexico)}

\section{ABSTRACT}

This descriptive research, which uses in depth interview, shows interactions between leadership styles and gender in 4 \& 5 Star-hotels in Tijuana, Mexico. The results show recognition of female leadership, as well as the relationship between gender stereotypes and transformational leadership as a consequence of care work. Women in managerial positions prefer a participative leadership style as a strategy to create alliances to avoid questions about their leadership capacity as well as work-life balance strategies.

Keywords: gender; leadership; hotels; Tijuana.

Fecha de recepción: 4 de marzo de 2017.

Fecha de aceptación: 17 de octubre de 2017.

Facultad de Turismo y Mercadotecnia. Universidad Autónoma de Baja California. Calzada Universidad no. 14418. Fracc. Mesa de Otay-Altabrisa.TIJUANA, B.C. México.C.P. 22420.E-mail: diaz.isis@uabc.edu.mx 


\section{INTRODUCCIÓN}

En México, el sector de turismo y viajes es uno de los que ocupa un importante número de mujeres (SECTUR-COLMEX, 2011). Dentro del total de empresas directamente vinculadas con el turismo, la industria hotelera es una de las más representativas (Dai, Chen y Zhuang, 2016; O’Neill, 2012) y no pocas veces ha sido considerada como la base de un turismo próspero. En México la industria hotelera aporta 9.1 por ciento al Producto Interno Bruto (PIB) Turístico (INEGI, 2015) y durante el 2014 generó 6.2 por ciento de los puestos de trabajo remunerados en el turismo (INEGI, 2016).

Debido a su importancia así como a la presencia de mujeres empleadas en la hotelería, se esperaría que dicha industria adoptara criterios amigables con el género. No obstante, diversas investigaciones han resaltado una división del trabajo basada en estereotipos (Fernández y Martínez, 2010; Burgess, 2003; Li y Wang, 2001; Chant, 1997), así como los efectos de ello, como la baja presencia de las mujeres en los puesto de toma de decisiones de los hoteles (Díaz-Carrión y Kido-Cruz, 2017; Samkangee y Dingani, 2013; OMT y ONU Mujeres, 2010), la presencia de techo de cristal y otras formas de segregación (Burgess, op. Cit.;Díaz-Carrión, Verján-Quiñones y Castrejón-Alonso, 2014; SECTURCOLMEX, op. Cit.).

El liderazgo es un concepto multicitado por diversas investigaciones realizadas en las últimas décadas; de acuerdo con Patiar y Lokman (2009) los estudios de liderazgo transformacional en hoteles son pocos y aquellos que además consideran el enfoque de género resultan más escasos (Marinakou, (2014).

Con la finalidad de identificar las posibles relaciones entre el género y el estilo de liderazgo en los hoteles de 4 y 5 estrellas de Tijuana (México) se recurre la investigación cualitativa, usando entrevistas en profundidad como principal herramienta metodológica.

Como principales hallazgos destacan la interacción entre los estereotipos y el estilo de liderazgo; así como otras barreras que limitan el acceso de las mujeres a las gerencias y terminan impactando también en el mantenimiento de los estereotipos de género. En el caso de las mujeres se encuentra más generalizada la aplicación del estilo transformacional como estrategia para superar los estereotipos de género, así como por motivos de desarrollo profesional y satisfacción personal. En el caso de las gerentes con personas a su cargo, el estilo de liderazgo transformacional se ha convertido en una importante estrategia para compatibilizar el trabajo con la familia y minimizar el conflicto entre ambas esferas.

\section{LIDERAZGO TRANSFORMACIONAL CON ENFOQUE DE GÉNERO}

Los primeros estudios de liderazgo aparecen a mitad del pasado siglo, y rápidamente se convierte en un concepto constante en las investigaciones organizacionales revisadas por la psicología, la sociología o la economía, por señalar algunas de las ciencias que prestan atención al estudio del liderazgo (Bass y Avolio, 1994; Burns, 1978; Yukl, 2013).

De acuerdo con Escandon-Barbosa y Hurtado-Ayala (2016:2-3), las teorías sobre el liderazgo pueden clasificarse aplicando los siguientes criterios: 1] en función de las características de quien ejerce el liderazgo, donde agrupan a la Teoría de Rasgos y las 
Teorías Conductuales; 2] en función de la situación, que comprende la Teoría de Roles y las Teorías de Contingencias y en función del Enfoque Integral, la cual a su vez comprende a las Teorías del Carisma y Teoría del Liderazgo Participativo.

De manera general, es posible decir que la evolución de las teorías va desde un concepto del liderazgo centrado en la figura del líder, independientemente de si el análisis se centra en quien dirige (Teoría de Rasgos) o la efectividad de la persona al momento de elegir un estilo determinado (Teorías Conductuales), hasta llegar a considerar al liderazgo ya no sólo limitado al líder, sino abrir dicho concepto a la consideración del rol jugado también por el equipo de trabajo; asociándose entonces al liderazgo con una conducta grupal (Teoría del Liderazgo Participativo) que evidencia la importancia del contexto de la organización y el sociocultural (ídem).

Por ello, la clasificación de estas autoras resulta útil para evidenciar el cambio de énfasis experimentado por el concepto de estudio; que pasa de centrarse en la persona que detenta o ejerce un liderazgo individual a enfocarse en uno colectivo, vinculándolo así con el contexto y por ende resulta sumamente útil para analizar el liderazgo transformacional desde una perspectiva de género.

Si bien el liderazgo tiene ya una trayectoria consolidada en los estudios organizacionales, la combinación de género y liderazgo como categorías de estudio se sitúa hacia finales del pasado siglo XX (Powell, 2011). Los primeros trabajos focalizan el género como el principal factor de diferencia para elegir un estilo de liderazgo, mientras que estudios más recientes señalan la necesidad de análisis centrados en las razones que conducen a las mujeres a optar por un estilo determinado. No obstante, en ambos casos se reconoce el papel de los estereotipos de género prevalecientes en diversas organizaciones y contextos sociales como un importante factor de elección (Marinakou, 2014; Medina-Vicent, 2015).

El concepto de liderazgo transformacional es introducido por Burns (1978) y desarrollado por Bass y Avolio hacia finales del siglo pasado (Bass, 1997; Bass y Avolio 1994); un liderazgo transformacional se distingue por influir en el equipo de trabajo a través de procesos de identificación e internalización (Bass, 1985), articulando una visión a largo plazo y siendo capaz de vincular intelectualmente a dicho equipo para encauzar los esfuerzos a la vez que se empatiza con el resto de quienes componen el equipo (Bass y Avolio, 1994; Yukl, 2013).

Para cumplir con ello se han identificado 4 dimensiones: 1] Motivación inspiracional, 2] Influencia idealizada, 3] Estimulación intelectual y 4] Consideración personalizada (Bass, 1997; Bass y Avolio, op. Cit.). A través de ellas, el liderazgo transformacional generaría nuevas ideas y soluciones a los retos impuestos por el dinamismo en los entornos organizacionales (Gumusluogy e Ilsev, 2009; Patiar y Lokman, 2009), al mismo tiempo que trabajan procesos de empoderamiento con el resto del equipo, lo cual les permite convertirse no sólo en excelentes colaboradores sino además en mejores personas (Minett, Yaman y Denizci, 2009). A partir de las interacciones de las cuatro dimensiones se generan procesos de interacción social entre quien lidera y el resto del equipo, lo que se constituye en la base del liderazgo transformacional (Tromp y Blomme, 2014:89). De manera esquemática, tanto características como beneficios de dicho estilo se pueden ver en la figura 1. 
Figura 1

ESTILO DE LIDERAZGO TRANSFORMACIONAL

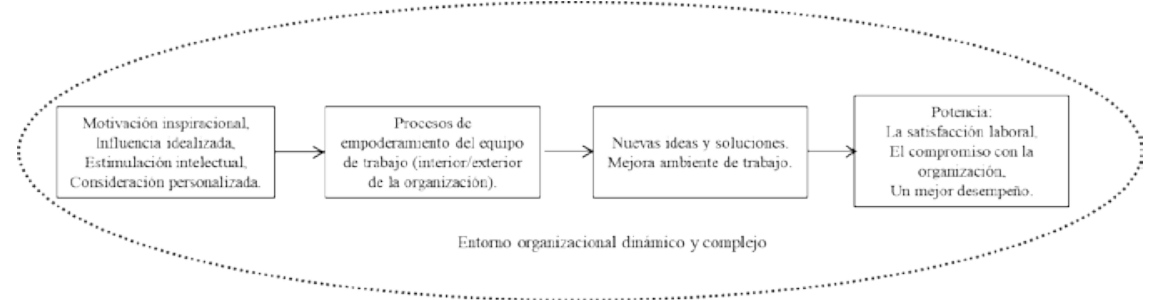

Fuente: Elaboración propia a partir de diversos autores.

Algunos autores, consideran que el liderazgo transformacional es el más adecuado para los hoteles debido al dinamismo y complejidad del sector (Tracey y Hinkin, 1994), aunque Salem y Kattara (2015) encuentran en su investigación en hoteles de 5 estrellas en Egipto que dicha relación puede variar dependiendo de las características del departamento o área del hotel. Por su parte, Patiar y Lokman (op. Cit.) señalan directamente que la mayoría de los hoteles aplican poco este estilo de liderazgo a pesar de los beneficios que pueden obtener, por ello promueven su inclusión en dichas organizaciones como un importante componente de competitividad en entornos dinámicos.

Precisamente, en este ejercicio de reconocimiento del dinamismo, se inscriben las propuestas de diversidad y sus interacciones entre las organizaciones y su entorno, siendo el género una de las múltiples variables a tener en cuenta en los estudios organizacionales en general, y de liderazgo en particular. Lo anterior debido a que, como señala Powell (2011) técnicamente las teorías de liderazgo no promueven la prevalencia de un género sobre otro, pero tampoco resultan neutras; ello debido a la fuerte recurrencia del uso de los estereotipos de género al interior de las organizaciones.

Los primeros estudios sobre liderazgo y género, de la década de los 1970 (ídem:3), se caracterizan por visibilizar la baja presencia de mujeres en puestos de liderazgo (Cuadrado, 2003) y en puestos socialmente considerados como adecuados para las mujeres (Eagly et al., 1995); algunos de esos primeros estudios evidencian el uso de diferentes estilos según el género (Rosener, 1990). También resaltan la importancia del contexto -tanto organizacional como social- y su estrecha relación con el estilo de liderazgo (Rosener, 1990; Maher, 1997); así, mientras que el liderazgo de los hombres es considerado más efectivo en las organizaciones militares, el de las mujeres lo es en las de tipo educativo, gubernamental y de servicios social (Powell, op. Cit.; Bass, 1985).

Estos hallazgos se convierten en punto de partida para considerar un estilo de liderazgo denominado "femenino" y caracterizado por la cooperación, la colaboración, el bajo control y la resolución de problemas recurriendo a la intuición, la empatía y la racionalidad (Loden, 1985 Loc. Cit. Cuadrado, 2003). La determinación de los modelos de liderazgo "femenino" y "masculino" se basa en un principio de diferenciación esencialista que si bien resultaba importante al momento de visibilizar la baja participación de las mujeres como líderes y la valoración de un estilo basado en la negociación queda, 
actualmente, superado ante una organización heterogénea y la necesidad de análisis del relevante papel de las dinámicas sociales al momento de optar por un estilo de liderazgo (Medina-Vicent, 2015).

Por ello resulta interesante el estudio de Bajcar et al. (2015:3675) quienes vinculan el estilo de liderazgo con el pensamiento estratégico, enfatizando el papel de múltiples variables organizacionales y extra organizacionales al momento de decidir por un estilo de liderazgo. Asimismo, Cuadrado y Molero (2002) hacen hincapié en la necesidad de abordar los procesos psicológicos subyacentes a la hora de adoptar un determinado estilo; toda vez que en su investigación encuentran diferencias poco significativas entre hombres y mujeres. La explicación de las diferencias, concluyen, puede deberse más a supuestos establecidos por las propias personas entrevistadas que al género. Lo anterior evidencia nuevamente la importancia del contexto, pues a partir de éste se definen los estereotipos de género y con ello las categorías sobre las que se construyen y validan las categorías, pero también la interacción de los estereotipos en el entorno organizacional.

En esta línea, trabajos más recientes proponen reflexionar sobre la necesidad de superar los estereotipos tradicionales que han sido -y son aún- usados para definir los estilos de liderazgo de las mujeres y los hombres. Así, Powell (op. Cit.) considera que los estereotipos son resistentes al cambio y señala la persistencia de masculinización en los estilos de liderazgo; toda vez que, no obstante, el incremento de mujeres en puestos gerenciales, tanto los hombres como las mujeres continúan definiendo un liderazgo efectivo recurriendo a características tradicionalmente validadas como masculinas (por ejemplo: la independencia, el riesgo o la agresividad).

También Medina-Vicent (2015) propone una revisión crítica para superar tanto la masculinización del liderazgo como el uso de esencialismos; pues considera que ambos han contribuido a difundir la cooperación, la empatía o la intuición como atributos exclusivos de las mujeres líderes. De igual forma, Lupano y Castro (2011) evidencian la necesidad de rebasar los estereotipos para así generar nuevas definiciones de estilos de liderazgo que, efectivamente, potencien la inclusión en entornos empresariales transformacionales independientemente del género (ibídem:27). Como señalan Lemoine et al. (2016) la presencia cada vez mayor de mujeres en las organizaciones, así como una tendencia al reconocimiento de aspectos tradicionalmente asignados a los hombres (como la independencia o el ejercicio del poder) y de aquellos atribuidos a las mujeres (como la empatía o la negociación) contribuye a generar nuevas interpretaciones sobre las definiciones y el ejercicio del liderazgo.

En general, los trabajos recientes proponen superar el uso de etiquetas "masculino" o "femenino" en los estilos de liderazgo; estas recientes propuestas evidencian la evolución de unos planteamientos teóricos que propugnan por una (re)construcción de las identidades de género, tanto en el entorno empresarial como en el resto de la sociedad. Generando con ello estilos de liderazgo efectivamente neutros al género, y capaces de valorar que el cuidado de los demás, la paciencia o la empatía no son sinónimo de femenino, ni dominio exclusivo de las mujeres; al momento que hacen lo propio también con aquellas características que tradicionalmente han sido validadas como exclusivas para los varones.

Los estudios de género en el turismo son pocos (Figueroa-Domecq et al., 2015) y más aún en lo referente a la industria hotelera y el estilo de liderazgo (Marinakou, 2014). 
Entre las primeras investigaciones destaca la de Maxwell (1997:233), quien recurre a una metodología cualitativa para entrevistar a gerentes generales de hoteles en Glasgow y Edimburgo; entre sus hallazgos destaca sus percepciones de una comunicación más cercana con sus equipos de trabajo, preocupación por sus colaboradores, cuidado en los detalles y en general una mayor empatía con su equipo -ajustando sus estilos de liderazgo a la situación-. Las gerentes entrevistadas consideran siempre estar disponibles para ser consultadas, contribuyendo con ello a promover el trabajo en equipo.

Otras investigaciones con enfoque de género que se han interesado, de manera secundaria, en el liderazgo son, por ejemplo, la Ng y Pine (2003:93), quienes en su investigación cuantitativa en los hoteles en Hong Kong, encuentran una mayor valoración de la comunicación efectiva, como elemento central del estilo de liderazgo, por parte de las gerentes que de sus colegas. Por su parte, la investigación cualitativa de Kattara (2005:246) en los hoteles egipcios, detalla la elevada presencia de las mujeres en las gerencias caracterizadas por una fuerte presencia de las actividades de trato al cliente y la descripción de estas áreas recurriendo a los estereotipos de género; así, las mujeres resultan idóneas para estos puestos por considerarse que son más empáticas y cuidan del huésped mejor que los hombres.

Mientras que Marinakou (op. Cit.:22), con su investigación cualitativa en los hoteles griegos, encuentra que las mujeres delegan más en su equipo que los hombres, comparten la toma de decisiones con su equipo, son más pacientes y amables, potencian a su equipo de trabajo, reconocen la posibilidad de equivocación y la importancia de reconocer los errores. Finalmente, Díaz-Carrión y Kido-Cruz (2017:212) constatan en su investigación cuantitativa sobre hoteles en Tijuana por un lado, el reconocimiento de la valía del trabajo de las mujeres, pero por otro un liderazgo más cuestionado que en el caso de sus colegas.

A partir de los hallazgos de los trabajos anteriores, es posible identificar un estilo de liderazgo de tipo transformacional, donde las gerentes promueven el diálogo, la cercanía, el soporte y la empatía con su equipo de trabajo; sin embargo, en ninguno de los casos se analizan las posibles razones que llevan a las gerentes de los hoteles a optar por este estilo, de ahí el interés de esta propuesta de contribuir en ello.

\section{METODOLOGÍA}

Esta investigación utiliza una metodología cualitativa para identificar las principales razones que sustentan la elección del estilo de liderazgo ejercido por las mujeres que ocupan las gerencias de nivel medio en los hoteles de 4 y 5 estrellas de la ciudad de Tijuana. Si bien el tema ha sido estudiado desde la metodología cuantitativa (Patiar y Lockman, 2009; Horstmeier et al., 2016), se considera que para el objetivo de este trabajo es más apropiada la metodología cualitativa por ser el liderazgo y el género fenómenos sociales (Marinakou, op. Cit.), por buscar un acercamiento más igualitario con la muestra de estudio y perseguir la comprensión del liderazgo desde una perspectiva social en las sociedades post modernas (Swirskya y Angelonea, 2015; Burgess, 2003); buscando con ello potenciar la interseccionalidad (Scholz, 2016; Wilson y Hollinshead, 2015) y promover sociedades más equitativas e igualitarias (Woodward y Woodward, 2015).

La principal herramienta utilizada es la entrevista en profundidad semi estructurada (Ayres, 2008:810-811; Dicicco-Bloom y Crabtree, 2006:315) y a manera de triangula- 
ción se recurre a las notas de campo (Leech y Onwuegbuzie, 2006:340). El guión de la entrevista se estructura en dos apartados generales; el primero busca obtener información socio económica de la persona y las generalidades de la función desempeñada en el hotel, mientras que el segundo se divide en dos sub apartados orientados a identificar el tipo de liderazgo y los beneficios obtenidos, así como las razones que llevan a dicha la elección.

Para la determinación de la población se recurre al directorio de la Secretaría de Turismo del Estado de Baja California (SECTURE) y se contacta con el Departamento de Recursos Humanos de los hoteles para obtener la totalidad de las gerencias en cada uno. La población de estudio son las mujeres y los hombres en puestos de gerencia general y gerencia media que se desempeñan en los hoteles de 4 y 5 estrellas de Tijuana, de acuerdo con información de campo se obtuvo un total de 77 gerencias de nivel medio para el total de los 15 hoteles que operaban en el destino.

La muestra de estudio intencional o de conveniencia (Sampieri et al., 2010) se estratifica para buscar una saturación estructural de los informantes de estudio (ver figura 2), buscando así abarcar la mayor cantidad posible de tipos polares (Valles, 2002).

\section{Figura 2 \\ CARACTERIZACIÓN DE LOS TIPOS POLARES PARA LA MUESTRA DE ESTUDIO}

\begin{tabular}{|c|c|c|c|c|c|c|}
\hline Género: & $\begin{array}{l}\text { 1) Hombre } \\
\text { 2) Mujer } \\
\text { 3) Otro }\end{array}$ & $\begin{array}{l}\text { 1) Gerencia general. } \\
\text { Nivel de Puesto: 2) Gerencia de nivel medio. } \\
\text { 3) Colaborador/a. }\end{array}$ & $\begin{array}{c}\text { Tipo de administración } \\
\text { del hotel: }\end{array}$ & $\begin{array}{l}\text { 1) De cadena. } \\
\text { 2) Independiente. }\end{array}$ & Ciclo de vida: & $\begin{array}{l}\text { 1) Sin hijos/as. } \\
\text { 2) Pareja sin hijos/as. } \\
\text { 3) Pareja con hijos/as. } \\
\text { 4) Sin pareja con hijos/as. }\end{array}$ \\
\hline
\end{tabular}

Fuente: Elaboración propia a partir de Valles (2002).

Las categorías de dicha estratificación son 1) género, 2) nivel de puesto, 3) tipo de administración del hotel y 4) ciclo de vida. Estos tipos polares han sido seleccionados a partir de los hallazgos de diversas investigaciones en las que, solos o de forma simultánea, aparecen como categorías relevantes (Marinakou, op. Cit.; Mooney y Ryan, 2009; PérezRamírez, Zizumbo-Villareal y Miranda-Contreras, 2012).

Dicha muestra se compone de un total de 27 mujeres y 10 hombres que colaboran en los hoteles de 4 y 5 estrellas de Tijuana, con dicho número se alcanza el nivel de saturación de la información. La etapa de campo se desarrolla entre los meses de noviembre del 2014 y marzo del 2015. Para la interpretación de los resultados se recurre al análisis de contenido con un enfoque narrativo (Ruiz Olabuénaga, 2012:194), siendo el género, el estilo de liderazgo, y el ciclo de vida las principales categorías.

\section{TIJUANA Y LOS HOTELES: GENERALIDADES}

La ciudad de Tijuana se ubica en el municipio del mismo nombre a $32^{\circ} 32^{\prime}$ de latitud norte y a $117^{\circ} 3^{\prime}$ de longitud oeste (INEGI, 2009:0). Sus colindancias son: al norte con los Estados Unidos de América (EEUU) y el municipio de Tecate (Baja California), al 
este con los municipios de Tecate y Ensenada (ambos de Baja California), al sur con los municipios de Ensenada y Playas de Rosarito (ambos también del mismo estado), al oeste con el municipio de Playas de Rosarito y el Océano Pacífico (ibídem).

De acuerdo con los datos del último Censo de Población y Vivienda 2010 (INEGI), la ciudad contaba con poco más de 1.55 millones de habitantes, de los cuales 49.7 por ciento eran mujeres (INMUJERES). A nivel nacional la media supone una población femenina de 51.16 por ciento, ligeramente por encima de la población femenina de la ciudad y también a nivel estatal (ídem).

Tijuana es una de las pocas ciudades mexicanas que presentan un índice de masculinización: por cada 100 hombres hay 99 mujeres (INMUJERES, s/f), este índice es incluso mayor al registrado en todo el estado bajacaliforniano (en un punto porcentual) y se explica como consecuencia de la fuerte tradición migratoria de la ciudad.

Esta ciudad fronteriza no ha quedado exenta del proceso de terciarización de la economía; según datos del Instituto Nacional de Estadística, Economía, Geografía e Informática (INEGI) en el año 2009 las actividades terciarias contribuyeron 63.22 por ciento del Producto Interno Bruto (PIB) Estatal. Y actualmente resulta un destino turístico que comienza a posicionarse a nivel nacional (Ruiz, Martínez y Verján, 2015; Bringas y Toudert, 2016); aun cuando la presencia de esta actividad dista mucho de alcanzar los niveles de otras regiones del país, la ciudad atrae cada vez un mayor número de turistas mexicanos y es un destino importante para la california americana (Bringas y Toudert, op. Cit.:114).

Según el registro de la Secretaría de Turismo del Estado (SECTURE) a la fecha están registrados un total de 6 hoteles de 5 estrellas y 9 hoteles de 4 estrellas en la ciudad. Ambas categorías de hoteles se concentran en dos ubicaciones; por una parte están los hoteles cercanos al Aeropuerto Internacional General Abelardo L. Rodríguez, y por otra los ubicados a lo largo de la denominada Zona Río, o en sus alrededores; ambas zonas resultan muy accesibles a la frontera con los EEUU.

Con respecto al tipo de administración del hotel (de cadena o independiente), se destaca que un 44.4 por ciento de los hoteles de 4 estrellas pertenecen a una cadena, mientras que este porcentaje alcanza el 50 por ciento para el caso de los hoteles de 5 estrellas.

\section{RESULTADOS Y DISCUSIÓN}

A continuación se presentan los principales resultados de la investigación. Primero, se contextualizan los puestos y/o departamentos en los hoteles de Tijuana y posteriormente se revisan los estilos de liderazgo y las razones que justifican su elección.

\subsection{Explorando la masculinización en el puesto de Gerencia General}

A partir de un primer análisis, se destaca la baja presencia de mujeres al frente de la gerencia general de los hoteles en el destino; de los 15 hoteles de 4 y 5 estrellas que existen en la ciudad, 3 reportan a una mujer ocupando dicho puesto; lo anterior es congruente con otros hallazgos (Li y Wang, op. Cit.:191; Kattara, op. Cit.:241; Yang, 2011:367).

La figura de la mujer al frente de los hoteles de 4 y 5 estrellas en Tijuana aparece como un hecho puntual; de acuerdo con la opinión de las personas entrevistadas la geren- 
cia general es un puesto masculinizado, independientemente de si se trata de hoteles de cadena o independientes. Al considerar que el destino tiende a ser más tradicional que otros (mujer, gerente de nivel medio, hotel de cadena, en pareja sin hijos; hombre, gerente general, hotel de cadena, en pareja con hijos; mujer, gerente de nivel medio, hotel independiente, sin hijos), existe la percepción de que los hoteles de cadena, principalmente aquellos pertenecientes a grupos nacionales o internacionales, presentan una mayor probabilidad de tener una mujer como gerente general. En este caso esta percepción se sostiene, pues 2 de las gerentes generales laboran precisamente en hoteles de cadena.

La ausencia de mujeres en las gerencias generales no se remite exclusivamente al momento puntual de la investigación, ninguna de las personas entrevistadas reconocieron la presencia de mujeres en estos puestos en el pasado: que yo recuerde, llevo 10 años en este hotel nunca ha habido una mujer en ese puesto (mujer, gerente de nivel medio, hotel de cadena, en pareja sin hijos). La principal razón de ello, en opinión de las personas entrevistadas, es la ausencia de interés: No les interesa y por eso no llegan (mujer, gerente de nivel medio, hotel independiente, en pareja con hijo). Esta aparente contradicción aparece también en las investigaciones de Maxwell (op. Cit.) y más recientemente en los trabajos de Ng y Pine (op. Cit.), así como en el de Marinakou (op. Cit.); si bien el análisis de esta percepción no forma parte del presente trabajo, no por ello deja de hacerse notar la asimilación de la presencia de las mujeres en los puestos de gerencia en los hoteles como un factor exclusivamente dependiente de su desempeño.

Lo anterior, sin considerar la, inevitable, relación de este factor con las esferas personal y doméstica de todas las personas, pero que al ser las mujeres las tradicionales responsables del trabajo de cuidados impacta más en sus carreras laborales. De igual forma, se obvia el impacto de la presencia de estereotipos de género en la carrera profesional de las gerentes de los hoteles. Las razones por las cuales las mujeres no se interesan por hacer una carrera profesional que, eventualmente, las conduzca hacia la gerencia general tienen, en opinión de las personas entrevistadas, a la familia como la principal causa: Incluso entre las que ya llegan a ser jefas de departamento o gerentes de área, cuando tienen hijos se deciden por la familia (hombre, gerente general, hotel de cadena, soltero sin hijos).

La familia como una elección excluyente aparece generalizada en el caso de las mujeres:

De momento, no...yo no podría ser gerente general de un hotel de la cadena porque me implica cambiar de plaza cada ciertos años, no haces tu carrera en un solo hotel y ite imaginas moverme con mi esposo y con mis hijos? Eso siempre pesa en las gerentes, también al momento de elegir en qué área desarrollarte... yo creo que las mujeres buscan más las áreas en las que no cambias de plaza (mujer, gerente de nivel medio, hotel de cadena, soltera con hija).

Tampoco tan explícita, pero igualmente vinculada con la cultura discriminatoria es la respuesta de uno de los entrevistados, un gerente de nivel medio de un hotel de cadena (en pareja sin hijos), quien considera: En los hoteles que he trabajado, siempre es un hombre 
[el gerente general] y si es así y se reúnen, yo pienso... que un hombre se siente más a gusto trabajando con otros hombres". La exclusión de las mujeres a espacios organizacionales o extra organizacionales aparece en distintas investigaciones como una limitante para el acceso de las mujeres a los puestos más relevantes de los hoteles (Mooney y Ryan, op. Cit; Díaz-Carrión y Kido-Cruz, op. Cit.)

Solo una de las personas entrevistadas reconoce abiertamente al machismo como una de las razones de peso para la baja presencia de las mujeres: Aquí no hay mujeres gerentes por el machismo... todavía tienes que elegir entre tu familia o tu carrera, considero que los roles no han cambiado (mujer, gerente de nivel medio, hotel independiente, soltera sin hijos). El resto de las personas entrevistadas, consideraron que hombres y mujeres reciben las mismas oportunidades en los hoteles, asegurando que las promociones dependen exclusivamente de los resultados de su trabajo.

La percepción del poder como un atributo masculino aparece como una constante en la investigación de Samkange y Dingani (op. Cit.); a la fecha, en Tijuana también el ejercicio del poder en los hoteles se encuentra masculinizado. En el ambiente hotelero de la ciudad si bien se reconoce el avance que han tenido las mujeres en las últimas dos décadas: Cada vez hay más mujeres trabajando en los hoteles y ya no están sólo como cocineras (hombre, gerente general, hotel independiente, en pareja con hijos), el reconocimiento de su liderazgo resulta incipiente.

Al respecto se presentan opiniones extremas sobre la consolidación del mismo, ya que algunas de las personas entrevistadas consideran que es sólo cuestión de tiempo (hombre, gerente general, hotel de cadena, casado y con hijos; mujer, gerente de nivel medio, hotel independiente, soltera y sin hijos); siendo esta una opinión no sólo compartida por hombres, sino también por las mujeres.

Sin embargo, si la familia continúa presente como una razón de peso y las mujeres la experimentan como una elección excluyente, el desarrollo de su liderazgo no se resolverá exclusivamente con el paso del tiempo, como bien anotó una de las entrevistadas: Considero que los roles no han cambiado [...] yo he podido ser gerente de área porque elegí no tener hijos, ni casarme y creo que por eso me ha sido menos difícil, he tenido que luchar contra otros estereotipos de machismo o de imagen, pero no con ese (mujer, gerente de nivel medio, hotel independiente, soltera sin hijos).

\subsection{Puestos feminizados en las gerencias medias de los hoteles de 4 y 5 estrellas}

Dejando de lado el puesto de la gerencia general, la revisión se centra en la presencia de las mujeres entre las gerencias de nivel medio. A partir de las opiniones se encontró que con el paso del tiempo, las áreas feminizadas han tendido a disminuir, como comenta uno de los entrevistados: Tengo casi 30 años trabajando en los hoteles de la ciudad y cuando inicié todos eran hombres, mujeres como gerentes de área... que yo recuerde... sólo si cuentas al Ama de Llaves...todos, todos los demás....hombres (hombre, gerente de nivel medio, hotel independiente, divorciado con hijo).

El acceso de las mujeres a los puestos de gerencia media de los hoteles de Tijuana comienza en las últimas dos décadas, y se presenta principalmente a través de la gerencia de recursos humanos; a la fecha, uno de los puestos percibido como feminizado: En la 
mayoría de los hoteles en los que he estado hay una mujer como gerente de recursos humanos" (mujer, gerente de nivel medio, hotel de cadena, en pareja sin hijos).

La explicación a esta situación es: Porque hay que tratar con las personas... y las mujeres son más....más como...cálidas...como que eso se les da bien (mujer, gerente de nivel medio, hotel independiente, soltera sin hijos). La gerente de recursos humanos aparece como una figura mediadora entre los colaboradores y sus propios jefes inmediatos: ...hay veces que a alguien le niegan un permiso [su jefe directo] y entonces viene conmigo para ver si yo puedo hacer algo para que le den permiso [...] constantemente estás mediando entre los colaboradores y sus jefes o los gerentes de área y el gerente general (mujer, gerente de nivel medio, hotel de cadena, en pareja sin hijos).

Una de las principales características del liderazgo transformacional es la capacidad de negociación; en el caso estudiado, ésta se relaciona más con las mujeres (hombre, gerente general, hotel de cadena, divorciado con hijos; hombre, gerente de nivel medio, hotel de cadena, en pareja con hijas); así, mientras los hombres detentan un liderazgo de acción, las mujeres son percibidas como predispuestas a la negociación antes de confrontarse con sus colaboradores, buscando llegar a acuerdos y crear alianzas (mujer, gerente de nivel medio, hotel de cadena, en pareja con hijos).

El buen trato y la paciencia (vinculados con la negociación) se manifiestan asimismo como principales razones para otro de los puestos feminizados en los hoteles estudiados: el de gerente de eventos y/o ventas. En opinión de las personas entrevistadas, las mujeres suelen ocupar ese puesto debido a que: Saben tratar con el cliente (hombre, gerente general, hotel de cadena, en pareja con hijos). En este caso, saber tratar con el cliente requiere de: Paciencia, mucha paciencia, yo veo que los hombres no la tienen, por eso entran al departamento [de ventas], pero no, no duran (hombre, gerente de nivel medio, hotel de cadena, en pareja con hijas).

Por su parte el puesto de ama de llaves no corresponde a una gerencia de nivel medio, pero sí es y ha sido uno de los puestos y área feminizada; no obstante ser reconocida como: Uno de los puestos más duros y pesados en los hoteles (hombre, gerente de nivel medio, hotel de cadena, en pareja con hijos) está feminizado. Para algunas de las personas entrevistadas esta feminización se debe a la naturaleza de las actividades que en él se realizan y que están muy relacionadas al trabajo de cuidados (mujer, gerente de nivel medio, hotel de cadena, en pareja sin hijos; hombre, gerente de nivel medio, hotel independiente, divorciado con hijas; hombre, gerente general, hotel de cadena, en pareja con hijos). No obstante hay quien considera que en su feminización influye que es: Uno de los puestos con sueldos más bajos del hotel (mujer, gerente de nivel medio, hotel de cadena, soltera sin hijos); por ello: los hombres, algunos que entran al hotel y no hay otro puesto se quedan allí, pero tan pronto pueden piden cambio o si no se salen [...] es muy pesado y no te alcanza (hombre, gerente de nivel medio, hotel de cadena, en pareja con hijas).

Como es posible identificar, la presencia de estereotipos de género en los hoteles del destino aparece como un aspecto clave en la generación de áreas o puestos feminizados de nivel medio (Chant, op. Cit., Ng y Pine, op. Cit.). Lo anterior, a la vez que el puesto de gerencia general se mantiene como uno masculinizado como sucede en la mayoría de las investigaciones (Maxwell, op. Cit.; Kattara, op. Cit.; Samkange y Dingani, op. Cit.). 


\subsection{Liderazgo transformacional y género}

Una vez presentadas las generalidades de los hoteles estudiados y revisadas las áreas y puestos feminizados de las empresas, se muestran los hallazgos relativos al estilo de liderazgo utilizados por las gerentes de nivel medio de los hoteles en Tijuana haciendo énfasis en las razones que sustentan dicha elección.

Como una de las principales razones aducidas para la elección del liderazgo transformacional, destaca la percepción de cuestionamientos que aún se mantienen en este sector y el papel que juega la elevada responsabilidad del trabajo de cuidados del grupo doméstico; en el caso de las mujeres con personas a su cargo aparece como la principal motivación para optar por un liderazgo transformacional.

Finalmente, debido a la importancia que revisten las acciones de formación del liderazgo, se indaga acerca de la existencia de espacios formales para el aprendizaje del mismo al alcance de las gerentes de los hoteles. El aprendizaje de ejercicio del poder es una estrategia coadyuvante para superar los estereotipos de género que pueden estar circunscribiendo el tipo de liderazgo elegido por las gerentes.

\subsubsection{Estilo de liderazgo transformacional: sus beneficios diferenciados por género}

De manera general, el estilo de liderazgo ejercido por las gerentes es uno caracterizado por la participación y orientado hacia la relación; mientras que en el caso de los gerentes entrevistados se encontró que no existe una predominancia de un estilo de liderazgo u otro, las personas entrevistadas consideraron que los gerentes aplican diversos estilos de liderazgo y que en los últimos años los hombres han comenzado a usar uno de tipo transformacional. Introduciendo medidas de participación con el resto de su equipo por considerarlo una forma más efectiva al momento de alcanzar los objetivos y además ser un estilo promovido desde la gerencia general o el corporativo de los hoteles (hombre, gerente de nivel medio, hotel de cadena, soltero y sin hijos; hombre, gerencia general, hotel de cadena, casado con hijos). Lo anterior es consistente con la investigación de Eisner (2013:28-29) quien reporta un importante énfasis por parte de los líderes tanto en las tareas como en las personas.

Como se comentó con anterioridad, entre las principales características del estilo transformacional se encuentra la empatía y la negociación; de acuerdo con Medina-Vicent (2015), la necesidad de negociación o la empatía no deben ser entendidas como exclusivas de las mujeres; pero en opinión de los entrevistados la necesidad de negociación se debe al carácter maternal de ellas: Las mujeres son como más cálidas, nosotros [los hombres] somos más como que... de acción (hombre, gerente de nivel medio, hotel independiente, en pareja y con hijos).

La definición de la empatía con el resto del equipo de trabajo aparece entonces como exclusiva de las mujeres como consecuencia de los estereotipos de género y con ello se corre el riesgo de feminizar este estilo de liderazgo y devaluar el esfuerzo por el hecho de estar más generalizado entre las mujeres que entre los hombres (ídem). Para evitarlo, se hace necesario el reconocimiento del éxito de las mujeres al momento de ejercer un liderazgo transformacional: Me parece que cuando la mujer es una jefa, cuando te dice 
algo que has hecho mal... no sé, es como que... te lo dice distinto, no te regaña, no te hace sentir mal como cuando tu jefe es un hombre (hombre, gerente de nivel medio, hotel independiente, divorciado con hijos). Y el esfuerzo que conlleva la generación de un buen ambiente laboral y desactivación de conflictos a partir del liderazgo transformacional, lo que contribuye a cambiar el estereotipo de cuidados inherente a su condición de mujer: Es porque las mujeres son como más sensibles al respecto (ídem), algo que aparece constante entre los gerentes.

Para los gerentes que aplican un liderazgo transformacional, el trabajo en equipo y la motivación de sus colaboradores les proporciona otras ventajas, en la mayoría de los casos personales: Me gusta trabajar en equipo, lograr avances en equipo (hombre, gerente de nivel medio, hotel independiente, divorciado con hijos); así como vinculadas a la carrera profesional: en los hoteles como este [de cadena] hay muchas posibilidades de ascenso, si haces las cosas bien, se te van abriendo nuevas posibilidades como... pasar a una mejor plaza y así, si yo tengo un buen equipo, un equipo que me responde, eso se, se valora mucho (hombre, gerente general, hotel de cadena, casado con hijos).

Si bien los gerentes recurren al liderazgo transformacional, en ningún caso esta elección se debe principalmente al trabajo de cuidados del grupo doméstico. Esta posibilidad no está entre las principales razones que les lleven a practicar un estilo de liderazgo transformacional: sí, es una posibilidad, por supuesto que sí, que también para eso, pero yo creo que eso más en el caso de las mujeres... (hombre, gerente de nivel medio, hotel independiente, en pareja y con hijos).

Antes de finalizar, también es importante hacer notar que la satisfacción personal es también otra de las razones que motivan a la elección del liderazgo transformacional: me gusta estar a gusto con el resto de mi equipo de trabajo (mujer, gerente general, hotel de cadena, casada con hijos). El reconocimiento tanto por parte de su equipo colaborador como del resto de la empresa justifica el que unas y otros busquen construir liderazgos transformacionales: cuando las cosas salen bien es trabajo de todo mi equipo, yo sé que yo estoy al frente, pero mi trabajo es de todos, todos contribuimos a hacer las cosas bien y con ello a que los otros departamentos también puedan hacer las cosas bien [...] claro que da gusto cuando te reconocen el esfuerzo hecho (mujer, gerente de nivel medio, hotel independiente, sin hijos).

Lo anterior resulta importante para superar la percepción errónea de unas mujeres poco interesadas en su carrera laboral. Igual que los hombres, también las gerentes reconocen ventajas del liderazgo transformacional y lo valoran por motivos profesionales, así como personales; ello independientemente del ciclo de vida en el que se encuentre la mujer (mujer, gerente de nivel medio, hotel independiente, soltera y sin hijos; mujer, gerente de nivel medio, hotel independiente, en pareja y con hijos).

\subsubsection{Liderazgo transformacional y el reconocimiento del liderazgo de las mujeres}

De igual forma, las mujeres entrevistadas consideran que el liderazgo transformacional contribuye no sólo al fortalecimiento de su capacidad de negociación sino también al reconocimiento de su liderazgo (mujer, gerente de nivel medio, hotel independiente, soltera y sin hijos; mujer, gerente de nivel medio, hotel independiente, en pareja y con hijos). 
Así, por ejemplo, las gerentes perciben que: Cuando eres mujer tienes más difícil demostrar que te has ganado tu puesto y si tienes hijos pues ya empiezan las dudas de si vas a poder o no (mujer, gerente de nivel medio, hotel independiente, soltera y sin hijos). En opinión de las gerentes dicho cuestionamiento puede: venir de tu equipo o de otros departamentos... de hombres, pero también de mujeres (mujer, gerente de nivel medio, hotel independiente, en pareja sin hijos); y considera la entrevistada que esta situación es más generalizada cuando se es una mujer: si hay cuestionamiento [en el caso de los gerentes] pasa muy pocas veces, como es normal que haya hombres gerentes, pues no, a las mujeres sobre todo si son de áreas donde sólo hay hombres se les mira [cuestiona] más.

Con la finalidad de evitar cuestionamientos sobre la capacidad de liderazgo, la mayoría de las entrevistadas buscan mantener buenas relaciones con el resto de su equipo de trabajo: No, no llegas y mandas, le explicas a tu equipo qué hay que hacer o por qué hay que hacerlo, yo me siento mejor si estoy apoyada por mis colaboradores (mujer, gerente de nivel medio, hotel independiente, soltera sin hijos); así como con el resto de los departamentos del hotel: A veces no es sencilla la relación entre la parte de montaje o el restaurante y otros departamentos, puede haber muchas confusiones por eso yo busco mucha comunicación para evitar problemas, que no crean que voy a darles órdenes sin ser su jefa (mujer, gerente de nivel medio, hotel de cadena, en pareja sin hijos).

La búsqueda de apoyos permite que las mujeres se sientan más seguras ante la toma de decisiones que conlleva el liderazgo, ellas no buscan una confrontación como primera opción pues entienden dicha decisión como una pérdida que puede generar problemas en un futuro: si hablas las cosas, aunque tengas que tomar decisiones en contra de alguien, tratas de hacerle ver que hiciste todo lo que podías por apoyarle pero que a veces no es posible, si la persona lo entiende es como... que has desactivado un problema a futuro (mujer, gerente de nivel medio, hotel de cadena, en pareja sin hijos).

Sin embargo, son conscientes de que en ocasiones el liderazgo implica tomar decisiones de manera poco participativa: tienes que actuar con mano dura (mujer, gerente de nivel medio, hotel independiente, en pareja y con hijos). Entre las gerentes entrevistadas una toma de decisiones poco consensuada es una posibilidad a la que recurren eventualmente; y si bien no evaden la confrontación directa, la usan: cuando no hay otra opción, pero no, no me gusta mucho aunque cuente con el apoyo de mi jefe (ídem).

Los gerentes, por su parte, no evitan confrontaciones directas y las asumen como parte del ejercicio cotidiano que supone ocupar una gerencia media y se sienten seguros de contar con el apoyo de su superior cuando existen este tipo de confrontaciones: las mujeres son más de hablar, de resolver así los problemas, y uno es más como de no dar tantas explicaciones (hombre, gerente de nivel medio, hotel independiente, divorciado y con hijos).

Sobre la negociación como estrategia para demostrar la capacidad de liderazgo, comentar que ninguno de los hombres entrevistados consideraron un requisito el recurrir a ella para ser considerado un buen líder: bueno, debes tener un buen desempeño, pero no...no creo que deba explicarlo (hombre, gerente de nivel medio, hotel de cadena, soltero y sin hijos). Tampoco los gerentes consideran la evasión de enfrentamiento como una estrategia para ganar apoyos (hombre, gerente de nivel medio, hotel independiente, soltero sin hijos; hombre, gerente de nivel medio, hotel independiente, en pareja y con hijos); 
si optan por un estilo transformacional esta decisión no se vincula a un reconocimiento de su capacidad de liderazgo como sí aparece en el caso de las gerentes entrevistadas.

Asimismo los gerentes consideran que el liderazgo de los hombres no es tan cuestionado como en de las mujeres: no, no hay tanto cuestionamiento creo porque es más... más normal (hombre, gerente de nivel medio, hotel independiente, divorciado y con hijos); la percepción de la socialización de la masculinización del poder en los hoteles es también reconocida por las mujeres tanto para las gerencias medias como en el caso de la gerencia general (mujer, gerente de nivel medio, hotel de cadena, divorciada con hijas).

\subsubsection{El estilo de liderazgo y la ausencia de políticas de conciliación de la vida personal, doméstica y laboral}

De acuerdo con los resultados, el estilo de liderazgo transformacional se presenta también entre algunos gerentes de los hoteles; no obstante, la motivación para recurrir a éste presenta diferencias. En el caso de las gerentes con menores a su cargo, la realización de trabajos de cuidados aparece como un importante condicionamiento al momento de optar por un estilo de liderazgo que potencia el trabajo en equipo: Cuando eres mujer tienes más difícil demostrar que te has ganado tu puesto y si tienes hijos pues ya empiezan las dudas de si vas a poder o no... por eso te apoyas más en tus compañeros (mujer, gerente de nivel medio, hotel independiente, soltera y sin hijos). Mientras que en el caso de los hombres, el cuidado de la familia no se reconoce como un factor clave en la elección del tipo de liderazgo ejercido (hombre, gerente de nivel medio, hotel independiente, soltero sin hijos; hombre, gerente de nivel medio, hotel independiente, en pareja y con hijos; hombre, gerente de nivel medio, hotel independiente, divorciado con hijos; hombre, gerente de nivel medio, hotel independiente, divorciado con hijas; hombre, gerente de nivel medio, hotel de cadena, en pareja con hijas).

Si bien en México el número de personas divorciadas con menores a su cargo se ha incrementado en las últimas décadas, la mayor parte del tiempo están bajo la custodia de la madre (INMUJERES); en el caso de los gerentes de hotel entrevistados, ya sea separados o divorciados con hijos menores, éstos aparecen como cuidadores puntuales, incluso cuando los hijos residen en la misma ciudad. De lo anterior es posible inferir que la cuota de trabajo de cuidados requerida por ellos es baja y de ahí la limitada influencia que ello genera en la maximización del tiempo para dedicar a los trabajos de cuidados. Además de lo anterior, otras principales razones que justifican un estilo de liderazgo transformacional tiene que ver con la necesidad de apoyo para el trabajo de cuidados, este estilo de liderazgo les permite a las mujeres contar con apoyos para llevar a cabo medidas de conciliación tendientes a minimizar el conflicto trabajo vs familia. Como así considera una de las entrevistadas:

Si un día, por decirlo, necesito ausentarme un rato para... para llevar a una de mis hijas al doctor o algo de....una situación de urgencia familiar, pues yo confío en que el resto del departamento podrá suplir mi ausencia... es por eso que me interesa que todo colaboren, que sepan los pendientes y cómo resolverlos, que mi ausencia no sea como que todo está parado (mujer, gerente de nivel medio, hotel de cadena, divorciada con hijas). 
Esta justificación aparece principalmente entre las mujeres que tienen terceros a su cargo (frecuentemente hijos, aunque también pudiera tratarse de quienes cuidan de los padres), así como entre las mujeres que planean tener hijos en un futuro:

Una de las razones por las que comparto casi todo lo que sucede en el Departamento con el resto de mis colaboradores es porque cuando tenga un hijo necesitaré de todo su apoyo. Tú sabes que los hijos pequeños requieren de mucho tiempo y ya no se le puede tener la misma dedicación [en el hotel] que cuando estás soltera o no tienes hijos (mujer, gerente de nivel medio, hotel independiente, en pareja sin hijos).

Mientras que entre los gerentes entrevistados la necesidad de conciliar los tiempos cotidianos no se entiende como un beneficio para aplicar un estilo transformacional: En mi caso, pues sí, tratas de compartir con los demás de... de los colaboradores cómo... cómo funciona el resto del Departamento, hacer como... como un trabajo, sí, más en equipo pues...y lo haces, yo en lo personal lo hago porque las cosas hay que hacerlas en equipo (hombre, gerencia general, hotel independiente, en pareja y con hijos). Tanto en el caso anterior, como en los restantes, el ejercicio del liderazgo transformacional se arguye como una estrategia desvinculada del resto de las esferas que componen la vida cotidiana (particularmente la esfera doméstica) y sus demandas.

Lo anterior aparece como una constante en el caso de quienes no supervisan el cuidado de terceras personas, tanto en el caso de los varones y las mujeres solteras y sin hijos, como en el caso de aquellos divorciados y/o separados pero cuyos hijos radican en otra ciudad y están bajo el cuidado de la madre. Para estas personas, el trabajo en equipo, característica del estilo de liderazgo transformacional. aparece, por ejemplo, un importante número de veces como una posibilidad de disfrute del tiempo libre: supongo que si mi equipo de trabajo está entrenado, pueden funcionar sin que yo esté encima de ellos todo el tiempo... y así puedo quitarme el hotel de la cabeza por un rato mientras no estoy trabajando (hombre, gerente de nivel medio, hotel de cadena, soltero y sin hijos), pero no para realizar trabajo de cuidados.

En el caso de las gerentes solteras o sin hijos, el trabajo en equipo también aparece vinculado a la posibilidad de tener menos carga de trabajo en el hotel: te permite descansar un poco (mujer, gerente de nivel medio, hotel independiente, soltera y sin hijos), y poder dedicarlo a otras actividades profesionales: yo estoy estudiando una maestría los fines de semana... pero cuando nazca mi hijo lo más probable es que lo dedique a él, por eso necesito un buen equipo de colaboradores (mujer, gerente de nivel medio, hotel independiente, casada y embarazada) o personales: puedo ir al gym o a veces me veo con mis amigas (mujer, gerente de nivel medio, en pareja y sin hijos).

En el caso de las personas entrevistadas, la mayor parte del trabajo de cuidados es dedicado a la pareja y a los hijos; y la dedicación de tiempo para cuidar de los padres aparece aún como una actividad puntual. Sin embargo, no hay que pasar por alto la fuerte probabilidad de que con el paso de los años el trabajo de cuidados de las ahora gerentes de los hoteles en Tijuana deba contemplar el cuidado de adultos mayores, con lo cual la cuota de cuidados podría mantenerse elevada todavía por un importante número de años.

Por ello se hace necesario el reconocimiento de las interacciones existentes entre las esferas privada y pública al momento de interpretar los hallazgos de investigación, de 
lo contrario se estaría invisibilizando la, todavía, fuerte carga de trabajo de cuidados en manos de las mujeres tijuanenses y el peso que ello puede tener al momento de optar por uno u otro de los estilos de liderazgo.

\subsubsection{Ausencia de espacios formales de aprendizaje para el ejercicio del poder}

La socialización del liderazgo es uno de los elementos centrales para su generalización entre las mujeres (Linehan y Walsh, 1999:266; Li y Leung, 2001:193), en el caso de las gerentes de los hoteles éstas suelen carecer de espacios formales en los cuales aprender y mejorar sus capacidades como líder. En su mayoría han aprendido a serlo empíricamente: Aprendes a prueba y error [risas] (mujer, gerente de nivel medio, hotel independiente, en pareja y embarazada).

De igual forma, los espacios de aprendizaje formal se muestran importantes para aprender los aspectos generales del liderazgo: ... en la carrera [universidad, o en las capacitaciones que te dan en el hotel, pero tengo conocidas de hoteles independientes que no tienen la posibilidad de capacitación y entrenamiento que tenemos aquí [en los hoteles de cadena nacionales] (mujer, gerente de nivel medio, hotel de cadena, en pareja y sin hijos).

Para el caso de las gerentes que laboran en hoteles de cadena de operación a nivel nacional, uno de los principales recursos para aprender a ser gerente son los cursos de formación para aquellas personas que desean desarrollar una carrera profesional en la cadena:

Cuando entras a trabajar, llenas un cuestionario y ahí se te pregunta si tienes interés en quedarte en la cadena y moverte de plaza, entonces junto con tu desempeño te van monitoreando y cuando hay oportunidad te mueven a un entrenamiento en otra plaza y cuando hay una posibilidad te la dan [...] a mí me han servido mucho esos entrenamientos para saber cómo tener un mejor desempeño en mi trabajo (ídem).

Ninguna de las gerentes entrevistadas pertenece a alguna asociación (nacional o local) que promueva la socialización del liderazgo; a pesar de que el establecimiento de redes formales de apoyo entre mujeres es una de las principales recomendaciones realizadas desde la academia, ninguna de las mujeres entrevistadas consideró necesaria la apertura y/o participación en espacios de este tipo.

Lo anterior puede obedecer a varias razones; por un lado, como se comentó anteriormente, el liderazgo se mantiene masculinizado en Tijuana a pesar de la presencia cada vez mayor de mujeres en las gerencias medias de los hoteles. Otra de las razones puede ser también el establecimiento de espacios informales donde las gerentes de nivel medio socializan el liderazgo:

Los hoteles que pertenecen a la Asociación [de hoteles en la ciudad] se reúnen una vez al mes, yo me reúno cada mes con las gerentes de ventas, y... esos espacios son para compartir información de cómo va la plaza... son reuniones de trabajo, no se discuten aspectos distintos, pero como en mi caso, la mayoría de las gerentes de ventas somos mujeres, luego siempre te quedas un rato después de la reunión y a veces discutes algunas cosas de tu equipo de trabajo... pero no son, no son como reuniones donde aprendas o compartas cómo ser una mejor jefa (mujer, gerente de nivel medio, hotel independiente, soltera y sin hijos). 
No obstante, incluso el número de estos espacios informales son menores para las gerentes de nivel medio que para sus colegas masculinos; en el caso de las mujeres también el uso y/o acceso a estos espacios aparecen condicionado por el trabajo de cuidados: sí, bueno, hay compañeros que se van a los partidos o como... conviven más fuera del hotel, pero yo en mi caso veo difícil eso: o voy con mis compañeros a su partido de futbol o voy con mi hija a su partido de futbol ¿no? por así ponértelo (mujer, gerente de nivel medio, hotel independiente, en pareja y con hijas).

Aun cuando la posibilidad de convivencia existiera, quedaría pendiente de superar el ambiente de compadrazgo que existe en los espacios donde conviven informalmente los gerentes de los hoteles, donde todavía: Los hombres se sienten más a gusto estando con hombres, a las mujeres no les gusta el futbol... (hombre, gerente de nivel medio, hotel independiente, en pareja y con hijos).

La ausencia de espacios formales para el aprendizaje del liderazgo y sus implicaciones en el futuro laboral restringe la formación de futuras líderes (Li y Leung, op. Cit.); pues como reconocen las gerentes entrevistadas, no existe una capacitación sobre cómo aprender a ser una buena líder (mujer, colaboradora, hotel independiente, soltera y sin hijos; mujer, colaboradora, hotel de cadena, casada y sin hijos).

\subsection{Implicaciones para la industria hotelera en Tijuana}

Derivadas de la presente investigación sobre el liderazgo con enfoque de género en los hoteles de 4 y 5 estrellas de Tijuana; se desprende lo siguiente:

El liderazgo como concepto social debe sostenerse en actitudes y conductas responsables, por ello resulta inaceptable la presencia de barreras -visibles o invisibles- que limiten el acceso de las mujeres a los puestos de gerencia general, así como la presencia de éstas en áreas o gerencias socialmente validadas para ser ocupadas por mujeres a partir de los estereotipos de género, pues con ello se está potenciando la discriminación.

El reconocimiento de las bondades de un estilo de liderazgo transformacional de momento, se encuentra diferenciado por género y por ciclo de vida. Las gerentes con personas bajo su cuidado recurren a él principalmente como una estrategia de conciliación y para minimizar el conflicto entre el trabajo y la familia; después aparecen otras razones que pueden ser compartidas por los demás gerentes con una baja participación en el trabajo de cuidados y que se relacionan con la carrera profesional y motivos personales.

El turismo es una actividad basada, precisamente, en los cuidados y por ello es una buena oportunidad para revalorar este trabajo ante la sociedad y las organizaciones. Se considera que lo anterior coadyuvará a la erradicación de los estereotipos de género en el trabajo con la finalidad de efectivamente hacer neutras las habilidades y competencias necesarias para el buen desempeño de quienes allí laboran. Y con ello garantizar la igualdad entre hombres y mujeres (establecido por Ley en México desde el 2006).

Por otro lado, es importante señalar que la industria hotelera se caracteriza por ser una de las que más tiempo consumen y, por ende, es considerada como poco amigable con la familia, lo cual la hace parecer poco atractiva al momento de atraer o retener al capital humano (McGinley, Hanks y Line; 2017; Deery y Jago; 2015). Toda vez que el conflicto del trabajo vs familia es uno de los más críticos para las mujeres que laboran 
en el turismo, se recomienda la concientización de las diferencias de género y de manera complementaria también se sugiere la implantación de medidas de conciliación en los hoteles, con la finalidad de hacer más atractivo el sector hotelero entre el capital humano y potenciar el compromiso y nivel de satisfacción del recurso humano a la vez que se promueve la calidad de vida de éste.

Todo lo anterior, permitirá un avance cualitativo en la participación de las mujeres y los hombres en la industria hotelera de la ciudad y podrá tener un impacto directo sobre su nivel de compromiso organizacional, así como en la promoción de liderazgos efectivos.

\section{CONCLUSIONES}

En los hoteles de 4 y 5 estrellas de Tijuana, el liderazgo de las mujeres ha tenido importantes avances; sin embargo, todavía se mantiene una importante interacción entre los estereotipos de género y el estilo de liderazgo. Para el caso de las mujeres suele usarse la imagen de cuidadora para justificar la elección del liderazgo transformacional que busca la participación, la negociación, el trabajo en equipo, la buena comunicación y el potenciar el buen trato de las personas.

La vinculación de los estereotipos de género con el liderazgo genera que la figura de cuidadora y proveedor continúen aplicándose en los departamentos de los hoteles; asimismo, la figura maternal tradicionalmente aplicada a las mujeres se traslada al estilo de liderazgo transformacional. Es importante hacer mención de ello pues se corre el riesgo de continuar con el uso de las etiquetas de lo "femenino" y lo "masculino" para aplicarlo sobre las mujeres y los hombres. Resulta necesaria la superación de estos criterios esencialistas y el justo reconocimiento del esfuerzo de las gerentes para construir con sus equipos de trabajo espacios que minimizan las relaciones de poder y maximizan el diálogo.

El optar por un estilo transformacional basado en el trabajo en equipo como una estrategia para liberar tiempo y energía para el trabajo de cuidados no aparece en el caso de los gerentes entrevistados y en cambio es altamente valorada por las gerentes. De igual forma, las gerentes recurren al liderazgo transformacional como estrategia ante la ausencia de políticas de conciliación de la vida personal, doméstica y laboral; esto se presenta de manera más evidente en el caso de las gerentes que tienen personas a su cargo, y es más puntual entre quienes no experimentan esa situación. Por ejemplo, los gerentes que se reconocen a sí mismos como líderes transformacionales lo hacen convencidos de que pueden liberar tiempo también, pero en ninguno de los casos este tiempo será usado principalmente para trabajo de cuidados.

Aunque en algunos casos también los gerentes opten por un estilo transformacional, resulta importante señalar otras razones que sostienen el ejercicio de este estilo entre las gerentes, quienes a través de la negociación buscan evitar conflictos, concretar acuerdos y formar alianzas con su equipo de trabajo, así como con los otros departamentos o áreas del hotel; esta necesidad obedece a la percepción de cuestionamientos sobre su capacidad de liderazgo. A diferencia de lo que perciben las mujeres, los gerentes se sienten a gusto con el poder y no se sienten cuestionados por ello, no eluden la confrontación, ni usan la negociación con esos fines. En los hoteles de 4 y 5 estrellas de Tijuana, la presencia de los hombres como gerentes está socializada y no es extraña, ni discutida su presencia en 
puestos de gerencias media y/o general; para ellos, la elección de un estilo transformacional obedece a otras razones (personales $\mathrm{u}$ organizacionales) pero en uno $\mathrm{u}$ otro caso desvinculadas con la esfera doméstica de sus vidas cotidianas.

Aunque la investigación tomó en cuenta el nivel jerárquico de los puestos (gerencias general-gerencia de nivel medio), una de las limitaciones de este estudio ha sido el no considerar el factor salario -y otras prestaciones- como un elemento potencial de la discriminación y sus efectos sobre la elección de un estilo de liderazgo.

De igual forma, a pesar de haber logrado el punto de saturación, se recomienda identificar más tipos polares (por ejemplo, de hombres con una fuerte cuota de trabajo de cuidados de su grupo doméstico) que pudieran enriquecer el estudio de la temática integrando, de igual forma, otras categorías de género y no sólo contemplando las binarias mujer-hombre.

También para futuras investigaciones se sugiere considerar lo anterior y replicar el estudio en otros destinos turísticos con la finalidad de comprobar el peso que pudiera tener el trabajo de cuidados en destinos de sol y playa o de turismo cultural más posicionados en el país.

\section{REFERENCIAS}

AYRES, L. (2008): "Semi-Structured Interviews", en The Sage Encyclopedia of Qualitative Research Methods (Vol. 1-2), Sage Publications, Thousand Oaks, pp. 810-11.

AVOLIO, B. J. y BASS, B.M. (1988): "Transformational leadership, charisma and beyond", en Emerging Leadership Vitas, Lexington Books, Lexington, pp. 29-49.

BAJCAR, B., BABIAK, J., y NOSAL, C. (2015): "When leaders become strategists. A new look at determinants of leadership styles through their relationship with strategic thinking", Procedia Manufacturing, 3, pp. 3669-3676.

BASS, B.M. (1985): Leadership and Performance Beyond Expectations, Free Press. New York.

BASS, B.M. (1997): "Does the transactional and transformational leadership paradigm transcend organisational and national boundaries?", American Psychologist, vol. 52 (2), pp. 130-139.

BASS, B.M. y AVOLIO, B.J. (1994): Improving organizational effectiveness through transformational leadership. Sage Publications. Thousand Oaks.

BRINGAS, N. y TOUDERT, D. (2016): "Satisfacción turística del visitante fronterizo en Baja California, México”, Región y sociedad, vol. 65 (28), pp. 109-147.

BURGESS, C. (2003): “Gender and salaries in hotel financial management: it's still a man's world”, Women in Management Review, vol. 18 (1-2), pp. 50-59.

BURNS, J.M. (1978): Leadership. Harper Row. New York.

CHANT, S. (1997): "Gender and tourism employment in Mexico and the Philippines", en Gender, en Work \& Tourism. Routledge. London, pp. 120-179.

CUADRADO, I. (2003): “¿Emplean hombres y mujeres diferentes estilos de liderazgo? Análisis de la influencia de los estilos de liderazgo en el acceso a los puestos de dirección”, Revista de Psicología Social, vol. 18(3), pp. 283-307. 
CUADRADO, I. y MOLERO, F (2002): "Liderazgo transformacional y género: autoevaluaciones de directivos y directivas españoles”, Revista de Psicología del Trabajo y de las Organizaciones, vol. 18 (1), pp. 39-55.

DAI, Y-D., CHEN, K-Y. y ZHUANG, W-L. (2016). "Moderating effect of world-family conflict on the relationship between leader-member exchange and relative deprivation: links to behavioral outcomes", Tourism Management, vol. 54, pp.369-382.

DEERY, M. y JAGO, L. (2015): "Revisiting talent management, work-life balance and retention strategies", International Journal of Contemporary Hospitality Management, vol. 27 (3), pp. 453-472.

DÍAZ-CARRIÓN, I. A. y KIDO-CRUZ, M.T. (2017): "La participación de las mujeres en la industria hotelera con enfoque de género: un estudio de caso en la ciudad de Tijuana (México)", El Periplo Sustentable, no 33, pp. 192-220.

DÍAZ-CARRIÓN, I.A., VERJÁN-QUIÑONES, R. y CASTREJÓN-ALONSO, B. (2014): "El sector hotelero desde la perspectiva de género: el "techo de cristal" en hoteles de 4 y 5 estrellas en Tijuana”, Revista Turydes: Turismo y Desarrollo, no. 7(0). Recuperado de: http:// www.eumed.net/rev/turydes/17/suelo-pegajoso.html (accesado el 23 de octubre del 2016).

DICICCO-BLOOM, B. y CRABTREE, B. (2006): “The Qualitative Research Interview”, en Medical Education, vol. 40 (4), pp. 314-321.

EAGLY, A.H., KARAU, S. J. y MAKHIJANI, M.G. (1995): "Gender and the Effectiveness of Leaders: A Meta-Analysis”, Psychological Bulletin, vol. 117 (1), pp. 125-145.

EISNER, S. (2013): "Leadership: Gender and Executive Style", SAM Advanced Management Journal, vol. 78 (1), pp. 26-41.

ESCANDON-BARBOSA, D.M. y HURTADO-AYALA, A. (2016): "Influencia de los estilos de liderazgo en el desempeño de las empresas exportadoras colombianas", Estudios Gerenciales, vol. 32 (139), pp. 137-145.

FERNÁNDEZ, M. y MARTÍNEZ, L. (2010): "Participación de las mujeres en las empresas turísticas privadas y comunitarias de Bahías de Huatulco, México ¿Hacia un cambio en el rol de género?", Cuadernos de Turismo, n ${ }^{\circ}$ 26, pp. 159-151.

FIGUEROA-DOMECQ, C., PRITCHARD, A., SEGOVIA-PÉREZ, M., MORGAN, N. y VILLACÉ-MOLINERO, T. (2015): “Tourism gender research: A critical accounting”, Annals of Tourism Research, vol. 52, pp. 87-103.

GUMUSLUOGLU, L. y ILSEV, A. (2009): “Transformational leadership, creativity, and organizational innovation", Journal of Business Research, vol. 62 (4), pp. 461-473.

HORSTMEIER, C.; BOER, D.; HOMAN, A. y VOELPEL, S. (2016): “The Differential Effects of Transformational Leadership on Multiple Identifications at Work: A Metaanalytic Model", British Journal of Management, vol. 28 (2), pp. 280-298.

INSTITUTO NACIONAL DE ESTADÍSTICA, GEOGRAFÍA E INFORMÁTICA (INEGI). (2016): "Estadísticas a propósito del Día Mundial del Turismo (27 de Septiembre). Datos Nacionales”. Aguascalientes, Ags.: INEGI. Recuperado de: http:// www.inegi.org.mx/saladeprensa/aproposito/2016/turismo2016_0.pdf (accesado el 16 de septiembre del 2017).

INSTITUTO NACIONAL DE ESTADÍSTICA, GEOGRAFÍA E INFORMÁTICA(INEGI) (2015): “PIB y Cuentas Nacionales”. Recuperado de http://www.inegi.org.mx/est/contenidos/proyectos/cn/tur/default.aspx (accesado el 21 de septiembre del 2017). 
INSTITUTO NACIONAL DE ESTADÍSTICA, GEOGRAFÍA E INFORMÁTICA (INEGI) (2010): "Baja California". Censo de Población y Vivienda 2010. Recuperado de: http://www3.inegi.org.mx/sistemas/mexicocifras/default. aspx src $=487 \&$ e $=2$ (accesado el 23 de octubre de 2016).

INSTITUTO NACIONAL DE ESTADÍSTICA, GEOGRAFÍA E INFORMÁTICA (INEGI): (2009): Prontuario de Información Geográfica Municipal de Los Estados Unidos Mexicanos Tijuana, Baja California. México, D.F., INEGI.

INSTITUTO NACIONAL DE LAS MUJERES (INMUJERES). s-f. Indicadores de Desarrollo Humano y Género en México 2000 a 2005. Baja California. México, D.F., INMUJERES.

KATTARA, H. (2005): "Career Challenges for Female Managers in Egyptian Hotels", International Journal of Contemporary Hospitality Management, vol. 17 (3), pp. 238-51.

LEECH, N. y ONWUEGBUZIE, A. (2006): "Field Data”, en In The Sage Encyclopedia of Qualitative Research Methods (Vol. 1\&2), Thousand Oaks, Sage Publications, p. 340.

LEMOINE, G. J., AGGARWAL, I. y STEED, L.B. (2016): "When women emerge as leaders: Effects of extraversion and gender composition in groups", en The Leadership Quarterly, vol. 27 (3), pp. 470-486.

LI, L. y LEUNG, R.W. (2001): "Female managers in Asian hotels: Profile and career challenges", International Journal of Contemporary Hospitality Management, vol. 13 (4), pp. 189-196.

LI, L. y WANG, R. (2001): "Female Managers in Asian Hotels: Profile and Career Challenges",en International Journal of Contemporary Hospitality Management, vol. 13 (4), pp. 189-196.

LINEHAN, M. y WALSH, J. (1999): “Senior Female International Managers: Breaking the Glass Border", Women in Management Review, vol. 14 (7), pp. 264-272.

LUPANO, M. L. y CASTRO, A. (2011): “Actitudes desfavorables hacia mujeres líderes. Un instrumento para su evaluación”, Summa Psicológica UST, vol. 8 (2), pp. 19-29.

MARINAKOU, E. (2014): "Women in Hotel Management and Leadership: Diamond or Glass?”, Journal of Tourism and Hospitality Management, vol. 2 (1), pp. 18-25.

MAHER, K. (1997): “Gender-related stereotypes of transformational and transactional leadership”, Sex Roles, vol. 37 (3-4), pp. 209-225.

MAXWELL, G. (1997): "Hotel General Management: Views from above the Glass Ceiling", International Journal of Contemporary Hospitality Management, vol. 9 (5-6), pp. 230-235.

McGINLEY, S. P., HANKS, L. y LINE, N.D. (2017): “Constraints to attracting new hotel workers: A study on industrial recruitment", International Journal of Contemporary Hospitality Management, vol. 60, pp. 114-122.

MEDINA-VICENT, M. (2015): “Women's Leadership: an essentialist concept?”, Journal of Feminist, Gender and Women Studies, vol. 2, pp. 69-72.

MINETT, D., YAMAN, H-R. y DENIZCI, B. (2009): "Leadership styles and ethical decision-making in hospitality management", International Journal of Hospitality Management, vol. 28, pp. 486-493. 
MOONEY, S. y RYAN, I. (2009). “A woman's place in hotel management: upstairs or downstairs?", Gender in Management: An International Journal, vol. 24 (3), 195-210.

NG, C. y PINE, R. (2003): "Women and Men in Hotel Management in Hong Kong: Perceptions of Gender and Career Development Issues", Hospitality Management, vol. 22, pp. 85-102.

O’NEILL, J. (2012): “Using focus groups as a tool to develop a hospitality work-life research study“, International Journal of Contemporary Management, vol. 24 (6), pp. 873-885.

ORGANIZACIÓN MUNDIAL DEL TURISMO (OMT), \& ONU MUJERES. (2010). "Informe mundial sobre las mujeres en el turismo 2010". ONU Mujeres, Madrid.

PATIAR, A. y LOKMAN, M. (2009): "Transformational leadership style, market competition and departmental performance: Evidence from luxury hotels in Australia", International Journal of Hospitality Management, vol. 28, pp. 252-262.

PÉREZ-RAMÍREZ, C., ZIZUMBO-VILLAREAL, L. y MIRANDA-CONTRERAS, S. (2012): "Incorporación al Turismo Rural y Transformación del Habitus en la Mujer Campesina de San Pedro Atlapulco, México", Rosa Dos Ventos, vol. 4 (2), 158-177.

POWELL, G. (2011): “The Gender and Leadership Wars”, Organizational Dynamics, vol. 40, pp. 1-9.

ROSENER, J. (1990): "Ways women lead”, Harvard Business Review, vol. 68 (6), pp. 119-125.

RUIZ, G., MARTÍNEZ, O. y VERJÁN, R. (2015): "Valoración de atributos culturales e históricos en la imagen promocional de la ciudad de Tijuana como destino turístico", El Periplo Sustentable, $\mathrm{n}^{\circ} 28$, pp. 31-58.

RUIZ OLABUÉNAGA, J. (2012): Metodología de la Investigación Cualitativa. Deusto. Bilbao.

SALEM, I. y KATTARA, H. (2015): “Transformational leadership: Relationship to job stress and job burnout in five-star hotels", Tourism and Hospitality Research, vol. 15 (4), pp. 240-253.

SAMKANGE, F. y DINGANI, S. (2013): "Beyond the Glass Ceiling a Gendered and Cultural Hospitality Management Discourse on the Advancement of Women Based on Integrated Research Paradigms", Tourism \& Management Studies, vol. 9 (1), pp. 20-27.

SAMPIERI, R., FERNÁNDEZ, C. y BAPTISTA, P. (2010): Metodología de la Investigación. McGrawHill. Lima.

SCHOLZ, T.M.L. (2016): "Beyond "Roaring Like Lions": Comadrismo, Counternarratives, and the Construction of a Latin American Transnational Subjectivity of Feminism", Communication Theory, vol. 26, pp. 82-101.

SECRETARÍA DE TURISMO (SECTUR) y COLEGIO DE MÉXICO (COLMEX). (2011): “Análisis económico del mercado laboral en el sector turístico: hacia una política pública para la igualdad entre hombres y mujeres". México, D.F. Recuperado de http://www.sectur.gob.mx/PDF/planeacion_estrategica/COLMEX_entregable\%20 final.pdf (accesado el 29 de septiembre del 2017).

SWIRSKYA, J.M., y ANGELONEA, D.J. (2015): “Equality, empowerment, and choice: what does feminism mean to contemporary women?", Journal of Gender Studies, vol. 25 (4), pp. 445-460. 
TRACEY, J.B. e HINKIN, T.R. (1994): "Transformational leaders in the hospitality industry", The Cornell Hotel and Restaurant Administration Quaterly, vol. 35(2), pp. 18-24.

VALLES, M. (2002): Entrevistas Cualitativas. Centro de Investigaciones Sociológicas. Madrid.

TROMP, D. M. y BLOMME, R.J. (2014): "Leadership style and negative work-home interference in the hospitality industry", International Journal of Contemporary Hospitality Management, vol. 26 (1), pp. 85-106.

WILSON, E. y HOLLINSHEAD, K. (2015): “Qualitative tourism research: Opportunities in the emergent soft sciences", Annals of Tourism Research, vol. 54, pp. 30-47.

WOODWARD, K. y WOODWARD, S. (2015): "Gender studies and interdisciplinarity", en Humanities Social Sciences-Palgrave Communications, 1, 15018-. https://doi.org/ DOI: $10.1057 /$ palcomms.2015.18

YANG, Y. (2011): “Gender and Engineering Career Development of Hotel's Employees in China", Procedia, vol. 1, pp. 365-71.

YUKL, G. (2013): Leadership in organizations. Pearson. Harlow. 